\title{
Degeneración corioepitelial después de un embarazo a término
}

\author{
FERNANDO MERA GONZALEZ \\ Jete de Clinica del servicio de Maternidad
}

Creo conveniente hacer un estudio somero del corioepitelioma antes de relatar la historia clínica de una paciente que estuvo hospitalizada en e! servicio del Profesor Rodriguez Apon. te (Instituto de Protección Materno-in: (antil), y a quien me correspondio observar y tratar en mi condición de Jefe de Clínica de la sala de Aislamiento.

Es el corioepitelioma una neoplasia de gran poder destructor y metastási. $c o$. constituída por elementos ectodérmicos de origen fetal, con prolifera. ción exagerada de células sincitiales y de Langhans, y que recuerda la es. tructura intima de las vellosidades coriales. Esta neoformación hace saLiente dentro de la cavidad uterina, y es de color rojizo con manchas azula. das, por la formación de espacios sanguíneos con trombosis y por la destrucción de las paredes vasculares, debidas al sincitlo proliferante. Penetran sus elementos en las paredes del útoro, hasta perforarlo algunas veces, $y$ de alli, la malignidad del-tumor, ya que estos tejidos normales prolifera dos aumentan la agresividad trípsica mientras disminuyen las delensas lo cales antitrípsicas de la decidua ma. ternc, que en el embarazo normal fre. nar: al sincitio $\gamma$ su ofensiva histolitica.
Fue Marchand, quien por primera vez enunció la naturaleza ectodérmica del corioepitelioma y lo diferenció fotalmente: a) de los deciduomas de Sanger, quien le atribuía naturaleza decidual de origen materno, y b) de los teratomas del testículo, que probablemente provienen del ectodermo fetal incluido en el teratoma conqeni. to. Hoy en día se puedé comprobar que la existencia de un corioepitelio. ma requiere, como condición previa, la participación de células tetales.

Ya aclarado el origen de esta neo. formación, es lógico suponer que ella aparece en la-mujer con gestación normal o anormal ya durante el em. barazo o bien después de evacuad. el contenido uterino. La mayor tre cuencia de esta neoplasia $(50 \%)$ acurr? después de los embarazos molares. por la transformación del ectodermo fetal, pues existe una similitud en su constitución anatómica, ya que en la mola vestcular o hidatiforme se halla una degeneración edematosa de las vellosidades con proliferación de las células epiteliales coriales, con edema de su estroma, que está sustifuido por una masa gelatinosa, y además, el proceso se retiene en la barrera de cldual, dejando el útero libre de los elementos ectodérmicos, conservande. 
hasta cierto punto, el cuadro de benignidad.

El estudio microscópico anatomopatológico de la neoformación en el corioepitelioma demuestra un sinnúmero de vasos trombosados, con abundancia de células redondas y leucoci. tos polimortonucleares, las células de Langhans y sincitiales proliferadas, y todo el estroma de la tumoración pro. fundamente vascularizado. En ocasio. nes el sincitio avanza hasta la luz ca. pilar, y los elementos epiteliales aparecen agrupados desordenadamente acusando múltiple carioquinesis y monstruosidades.

Como tumor maligno que es, da diversas metástasis, mas prefiere localizarse en la vagina por implantación superficial, e invade al pulmón, hígado, bazo y encéfalo por trasporte sanguíneo de las células carcinomatosas.

La sintomatología del corioepitelioma se sintetiza en: a) metrorragias, que pueden ser erráticas, frecuentes 0 repetidas, de mediana intensidad 0 profusas y espaeiadas, y qua aparocen en la mujer que ha tenido embara. zo normal, un aborto eutóplco o un aborto molar. La persistencie de hemorragias, rebeldes al tratamiento hemos tático y útero-tónico, agota a la enferma y pone de presente lenómenos anémicos, con palidez de la piel $y$ conjuntivas, subictericia, albuminuria, edemas maleolares y de los párpadoi, es decir los signos claros de caquexia: b) leúcorrea tétida y maloliente por la desintegración de los tejidos alterados, $\mathrm{y}$ c) dolor, que es inconstante $\mathrm{y}$ aparece cuando hay propagación del contenido uterino a la cavidad peritoneal

En la evolución de esta entidad har a veces temperaturas discretas, debtda a los procesos infecciosos por la al- teración de los tejidos carcinomatosos, cuyo medio es propicio para estas infecciones sobreagregadas. Al tacto vaginal, combinado con la palpación, se aprecia un ítero blando, aumentado de tamaño, con su orificio cervical entreabierto y permeable, que da salida a los tejido: modificados, fácilmente confundibles con restos placentarios u ovulares, y que inducen, en no pocas ocasiones, a practicar legrados uterinos por el convencimiento equivocado de ser restos retenidos de una gesta'íi. Por lo tanto siempre es necesario, en casos como este, enviar el producto del legrado al examen microscópico, para que su estudio aclare en detintiva el diagnóstico clínico.

$I_{2}$ demás, y esto es muy importante. nn los casos probables de degeneración corioepitelial, deben practicarse renciciones de laboratorio, de Friedmann y Gailli-Mainini, que hoy en dia sen las más usadas. Preciso es recordar que el corioepitelioma modifica las rec:cciones elevando y sosteniendo las títulaciones después de un aborto eutopico o ectópico o de un embarazo rormal. Después del aborto molar la excreción hormonal se negativiza en el término de 7 a 10 días, pero si los restos molares ya provocaron altera. ciones epiteliomatosas, la eliminación hormonal persiste con aumento progresivo.

El-truinimento de la neoformación epiteliocorial, una vez establecido el diagnóstico, consiste en la ínmediato extirpación del cuerpo y cuello uteri nos por vía vaginal o abdominal; lo ovarios pueden conservarse si no s. encuentran invadidos por la neopld sia, tenómeno apreciable durante acto quirúrgico. Los quistes luteinico regresan después de la extirpación de 
órgano degenerado que les dió origen. La radioterapia únicamente se aconso ja cuando es peligrosa la histerecto mía total, o si el corioepitelioma tiene metástasis varias. El tratamiento palig tivo se reduce entonces a mejorar lu mutematologic uterine mientras sobre vis la muerte.

Li) exito del tratamiento depende de Ia precocidad en el diagnóstico; y de la extirpación del tumor antes de pro ducirse la metástasis. Las estadisticas recientes de Payne arrojan resultados curativos en el $95 \%$ de los casos, sobre un total de 36 observaciones.

Debo aclarar que, después del tratamiento quirúrgico, conviene continuar la investigación biológica perió dica para comprobar el resultado tera peutico. En caso de metástasis persis la eliminación hormonal alta aunque no haya sintomatología clínica; si ha; curación se negativiza la reacción rápidamente.

Hecha la descripción del diagnóstico, sintomatología y tratamiento del corioepitelioma, paso a relatar la his. toria clinica correspondiente.

Nombre: M. del C. M. Edad: 22 años.

Eceha de ingreso: agosto 2 de 1949 Fecha de salida: agosto 19/49.

Historia No 1.583 de 1949. Servicio del Prolesor Rodríguez Aponte. Sala general.

Examen obstétrico: Ultimas reglas: octubre $30 / 48$.

Tensión arterial: $13.5 \times$ - 8 . Pulso, 84 .

Hora en que inició trabajo: agosto $2 / 49$ a las 6 a. $\mathrm{m}$.

Marcha de las contracciones uteri nas desde su iniciación: irregular. Estado de las membranas: intactas. Se creción vaginal: muco-sanguinolenta.
Inspección: estado general de la en. fermá: bueno.

Senos: calostrorrea, aréolas secun. darias:- Desarrollo del vientre: embarazo de 9 meses. En trabajo.

Organos genitales externos: normales.

Palpación: altura uterina, 30 centmetros. Consistencia de la matriz: nor mal. Situación longitudinal. Forma glo bulosa. Superficie regular. Contraccio nes uterinas en el momento del examen: frecuencia, cada 15 minutosi duración 30 segundos. Intensidad regular.

\section{Datos fetales:}

Feto único. Situación longitudinal. Presentación cefálica de vértice defle jada. Posición izquierda. Altura de ia presentación: insinuada. No hay des proporción cefalopélvica.

La paciente dice haber tenido un dolor fuerte el 2 de agosto a las 7 y $\mathrm{mo}$ dia, seguido de hemorragia vaginal discreta, y deló de sentir movimien. tos ietales.

Auscultación: ruídos cardiacos tetales negativos.

Tacto vaginal (agosto $2 / 49$ a las 12 y 20 horas): Vulva y vagina normales. cuello blando, borrado casi completa mente con dilatación de $1 \frac{1}{2}$ centime tros. Membranas íntegras.

Perto: goosto $3 / 49$. Hord: 5 y $5 \mathrm{a}-\mathrm{m}$.

Estado del niño al nacer: normal, anatómicamente.

Alumbramiento: espontáneo. Peso de placenta: 720 gramos. Membranas completas. Hemorragia no. Longitud del cordon: 35 centímetros.

Niño: número único. Sexo temienno. Peso 2.880 gramos. 


\begin{tabular}{|c|c|c|c|}
\hline $\begin{array}{l}\text { ULRPERIO: } \\
\text { Fecte }\end{array}$ & Senos & Alture ateriae & Lequilos \\
\hline VIII-3 & B. & 17 & Hemorrágicos \\
\hline VIII-4 & B. & 19 & Hemorrágicos \\
\hline VIII-5 & B. & 12 & Sangrientos \\
\hline VIII-9 & B. & 13 & Purulentos \\
\hline VIII-12 & B. & 7 & Serosangrier \\
\hline
\end{tabular}

Se suministraron durante el puer perio los siguienies medicamentos: 90 tas involutivas, sulfadiazina, 30 table tas, tintura de belladona. XX gotas? veces al día.

Cuadro térmico: puerperio sub-fe bril.

El estado de la paciente en la lecha de alta es satisfactorio.

La paciente reingresa el 16 de sep. tiembre/49 y queda hospitalizada en el servicio de Aislamiento dèl Profesor Moilca. Historia N 1.669 de 1949.

Se hace diagnóstico de puerperio tebril. Se le administran los siguientes medicamentos: penicilina $1.000 .000 \mathrm{~d}$ unidades, sulfadiazina, 30 tabletas; extracto hepático inyectable, cápsulas férricas, y sale por mejoría el día 4 de octubre/49.

Cuadro termico: temperaturas osci. lantes entre 39 y 36.5 grados.

La paciente reingresa al servicio dal Profesor Acosta, sala general, el día 14 de octubre/49.

\section{Historia No 1.887 de 1949.}

Diagnóstico: Retención de cothledo nes placentarios. Anemia intensa.

Pulso 100 p. m. Tensión arteriat, $9 \times 5$.

Se copia de la historia; La pacient: tuvo su primer parto hace dos meses en el servicio del Protesor-Rodrigue: Aponte; salió a los 10 días en condicio. nes satisfactorias. Reingresa a los 10 días -al serviclo de aislamiento por he- morragia abundante y dolores del bajo vientre. Allí la ponen en reposo y salió a los 20 días habiendo cesado las hemorragias.

Hace cinco días experimenta nuevamente hemorragia genital y dolores del bajo vientre. Llega al servicio a las 22 horas.

Tacto vaginal: vulva y vagina nor males. El cuello uterino se encuentra abierto y hacen hernia a través del orificio cervical los restos placentarios. No se pueden extraer digitalmnte.

Se le aplica ariestesia general y se extraen con pinzas de cuerpos extraños.

Se aplica ginergeno, coramina, solución dextrosada al $10 \%, 500$ c. c. con vitamina $\mathrm{K}$.

Et cuadro térmico de esta paciento durante el tiempo de su hospitalización en este servicio osciló entre 39 grados (después de la intervención) y 36 grados.

Sale por mejoría en octubre 26/49.

Se le suministró como medicación post-operatoria: cápsulas férricas, ex tracto hepático y sulfadiazina.

En noviembre $17 / 49$ se hospitaliza en el servicio del Profesor Rodríguez Aponte. Sala de Aislamiento.

Historia N $\mathrm{N}^{\circ} 2.369$.

En esta ocasión la paciente no da datos de sus hospitalizaciones anferio. res en el Servicio de Maternidad. La paciente da los siguientes datos: menarquila a los 16 años. Ciclo $30-3$ Menalgia. Ha tenido-flujos con sangre. Antecedentes obstétricos: un embara zo a término con feto muerto, hace tres meses.

Enfermedad actual: se quela de dolor abdominal, cefalalgía, vómitos, diarrea, hemorragia vaginal, tos sin 
expectoración. Se ordena: examen d orina, pues hay puntos ureterales do. lorosos.

Se establece un tratamiento a bas? de antibióticos (penicilina estreptomicina) y tónicos generales extracto he pático, cápsulas térricas).

Noviembre 21/49: So queja de dia trea abundante. Tratamiento: sulfasu xidina, atrinal.

Noviembre 23: hay hemorragia vaginal abundunte. Tratamiento: ergotrate, dos tabletas - Ginergeno una ampolla- Sulfadiazina.

Noviembre 29: Tacto vaginal: vulva y vagina normales. Cuello borrado, dilatado tres centímetros. Se aprecia la salida de restos ovulares por el orificio cervical. Cuerpo de la matriz blando, en posición normal, ligeramente aumentado de tamaño correspondien. do a un embarazo de dos meses. Diag. nóstico: Aborto incompleto de dos meses.

Tratamiento: Raspado uterino y ta. ponamiento, bajo anestesia con Pen. tothal sodico.

Noviembre 30/49: Se le extrae la mecha del taponamiento. Se le pres. criben antibióticos (penicilina-estreiptomicina) Tónicos generales, uterató nicos.

Diciembre 3/49: Continúa la metro ragia. Tensión arterial: $9 \times 3$.

So toma biopsia de endometrio y se lo ordena reacción de Friedmann cuan. titativa.

Transfusión de sangre total: 500 c. c.

Diciembre 4/49: continúa la hemorra. gia-váginal. Mal estado general. Ten. sión arterial: $9.5 \times 3$. Taquicardia, soplo sistólico de origen anémico en todos los focos de auscultación cardíaca.
Tratamiento: solución dextrosada al $5 \% 1.000$ c. c. Corti-renal una ampolleta. Normoclotín -Vitamina K - Extrac. to hepático inyectable y calor perma. nente.

Diciembre 10/49: Disminuyó la he morragia vaginal. Mejor estado gene. ral.

Diclembre 12/49: Continúa disminuyendo la metrorragia. El resultado de la radiogratia de vía biliares llega con diagnóstico de exclusión vesicular.

Diciémbre 13/49: El resultado de biopsia de endometrio pone de presente elementos de inflamación. La he morragia ha disminuido.

Sé establece diagnóstico de endometritis, post-aborto.

Diciembre 14/49: Contestan a la reacción biológica de Friedmann quo se había pedido, en los siguientes términos: Imposible hacerla por falta de conejos".

Diciembre 15/49: No hay hemorrugia vaginal. Leucorrea clara.

Diciembre 19/49: Aparece una túmefacción en la raíz del muslo derecho que es inflamatoria

Diciembre 21/49: Punto cístico muy doloroso. Aumenta el flujo vaginal. Pulso $115 \mathrm{p}$. m. Tensión arterial $9 \times 0$. Tos sin expectoración. Temperatura: 39.2 grados.

Se formula antibióticos y tónicos generales.

Diciembre 23/49: Mal estado general. Dolor epigástrico y en el hipocon. drio derecho. Vómito. Leucorrea abundante.

Diciembre 30/49; Hemorragia vagi. nal abundante, con coágulos y dolo. res tipo cólico. Vómitos. Dolor en punto vesicular. 
Enero 2/50. Tos con expectoración hemoptoica. La matriz se palpa dura y dolorosa. Leucorrea abundante.

Enero 10/50. Pulso-140. Tensión arterial, $9.5 \times 0$. Abdomen muy doloroso. Continúa el vómito. Como los datos clínicos denotan que hay un tumor ute rmo maligno y la metrorragia conti. núa, agravándose el estado general so resuelve prepararla para posible histerectomía. So pido transtusion san guínea pero es imposible practicarla por el $\mathrm{mal}$ estado de sus venas.

Enero 12/50. Empeora el éstado general -y se aplaza la intervención debido a las malas condiciones en que se halla la paciente. Tensión arterial, $6.5 \times 2$. Tiene retención de orina. Continúan el vómito y la hemorragia vaginal. Abdomen muy doloroso.

Enero 13/50. Muere la paciente y se envía su cadáver al laboratorto de Anatomía Patológica con el fin que se Ie practique una autopsia. Lleva diag. nóstico posible de tumor uterino maligno.

El cuadro térmico marca temperaturas oscilantes entre 39.5 y 37 grados durante el thempo de su hospitalización hasta la muerte.

\section{EXAMENES DE LABORATORIO}

Flujo vaginal: Examen bacteriológico. (Dbre. 20/49).

Diplococos gram negativos morioiógícamente semejantes al gonócoco, extracelulares.

\section{Análisis parcial de orina}

(Enero 11/50). Albummer: ligaras huellas Sedimento uratos amorfos $+t+7$. Leucocitos t.
Resultado anatomopatológico de la biopsia. Tejido órgano o región: Endometrio. Descripción macroscópica: Varios fragmentos de tejido blando color gris rojizo. Dimensiones: IxIxO,2 centimetros.

Diagnóstico microscópico: Inflamación aguda. El material enviado para examen está compuesto por tejido. aparentomente conjuntivo, en estado de completa desintegración. Se ven depósitos de fibrina $y$ de pus. Hay áreas de hemorragia.

(Fdo.) El Jele de la Sección: Sánchez Herrera.

\section{Diciembre $12 / 49$.}

\section{Análisis parcial de orina}

(Noviembre 19/49). Densidad: 1.015. Reacción ácida. Albúnima: muy ligeras huellas.

Sedimento: leucocitos $+i$ células epitelíales + .

Resultado anatomopatológico de la autopsia: *Tejido órgano o región: Pul. mones. Descripción macroscópica: hay adherencias intimas entre las dos hojillas pleurales y de éstas a la reja costal, al diafragma, al pericardio y al mediastino. Pulmón derecho: peso. 425 gramos. La superficie es de color gris rosado pálido y muestra conges. tión en la base, lo cual se confirma al corte. En el lóbulo superior se aprecian dos nódulos de consistencia firme, de 3.6 centímetros de díámetro el mayor de ellos que al corte presenta una zona exterior de color pardo obscura y una zona inferior de color rojo. En uno de éstos se ve una cavidad central de contenido purulento. Pulmón izqulerdo: 
peso 350 gramos. Con caracteres semejantes a los del derecho y con un nódulo de 3.8 centímetros de diámetro de aspecto carcinomatoso semejante a los descritos en el pulmón derecho.

\section{Diagnóstico microscópico: Metástasie} corio-carcinomatosa. Los certes pta. incados sobre los nódulos descritos matomicamente, dejan ver áreas ex. wusas de necrosis $y$ de hemorragic: rodeadas por zonas de reproducción de un neoplasma maligno, especialmente rico en vasos sanguíneos y cuya estructura recuerda de manera muv imperfecta, la de la placenta. Los ele mentos neopiásicos son hipercromáticos, de tipo Langhans los más y sincj. tiales otros. Los nódulos están rodeados por parenquima-pulmonar que presenta zonas de neumonia. En otros campos hay enfisema $y$ antracosis moderada.

Tejido, órgano o región: Higado. Descripción macroscópica: Peso 1.525 gramos. La viscera se encuentra bañada por líquido purulento viscoso fé 1ido, de color amarillo y con numero sas lalsas membranas. Este líquido en contidad de 1.050 c. c. baña todas las - visceras abdominales y pélvicas. La cápsula propia se encuentra cubierta de falsas membranas y presenta ad. herencias intimas a las visceras vec:nas. Al corte el parenquima es semifirme, de color carmelita claro y con arquitectura poco aparente. La vesícula biliar contiene escasa cantidad de barro biliar. Vías billares perméables.

\section{Diagnóstico microscópico: Hepatits} sub-aguda. El estroma muestra infiltractón leucocitária mono y polinuclear. Los vasos centrales están dilatados y también los capilares vecinos. En todos los campos se ve degeneración grasosa de la célula hepática. En el perituneo hay edema abundante acompeñado de infiltración densa por leucocitós polinucleares. La vesicula biliar muestra cambios post-mortem. Tejido: Organo o región: Riñones. Descripción macroscópica: Derecho, peso, 100 gramos, espesor de la corteza. 0.6 centimetros. La superficie exterior es de color rojo sombreado. La cár sula se desprende con tacilidad. A! corte es de consistencia firme, de color rojo amarillento $y$ de arquitectura bien aparente. Izquierdo, peso, 110 gra. mos. Espesor de la corteza 0.9 centime tros. Es de color rojo obscuro y la cápsula bien adherente. Al corte es de consistencia firme y muestra un pareñquima hemorrágico.

\section{Diagnóstico microscópico: Netritis} crónica. Se ve esclerosis moderada de los glomérulos. Los vasos sanguíneos están muy dilatados. Con frecuencia se aprecian cllindros hialinos. No es posible dar más detalles histológicos debido a que hay extensa desintegra. ción post-mortem.

Teiddórgano o región: Utero. Descripción macroscópica: Peso, $428 \mathrm{gra}$. mos. Dimensiones: $10 \times 9 \times 8$ centime tros. Tanto el miometrio como el endo metrio están reemplazados por un tejido blando de color rolizo con abundante áreas de necrosis. Este proceso ha afectado también al cuello uterino. La serosa se encuentra espesa y encapsula completamente al órgano. So bre el fondo, hacia la región izquierda y dos centímetros por encima de la im. plantación de la trompa correspon. diente se aprecia un orificio antractuoso de bordes irrégulares de 2.5 centí. metros de díametro por el cual emer. ge hacia la cavidad pélvica el teltdo intra-uterino. En la cavidad vaginal se 
encuentra líquido purulento del mismo aspecto del que se encontró en la cavidad peritoneal.

Diagnóstico microscópico: Corio-carcinoma: Los cortes practicados sobre e. fondo, muestran un neoplasma ma. higno de origen placentario, enorme mente intiltrante que invade los vases sanguineos y que se acompanic de grandes áreas de necrosis, en ocasio nes con formación de pus. Los elemen. tos que lo forman son hipercromáticos de tipo Langhans unos y otros, crgrupados formando sincitio. Todos estos elementos presentan alguna tendencia a agruparse formando pseudovellos:dades de estroma muy escaso. En ei cuello hay dilatación muy marcada de los vasos sanguíneos e infiltración leu cocitaria mononuclear.

El Jefe de la Sección.

\section{(Fdo.) M. Sánchez Herrera}

\section{Enero 26/50.}

La historia clínica nos enseña que si es posible la degeneración corioepite. hal en nuestro medio, $y$ que si las pu. blicaciones al respecto y las estadísticas propias nada nos indican, no por ello debemos concluir que esta entidad es extraña en Colombia, sino que. por el contrario, debemos esforzarnos por un mejor estudio clínico de esta alección; con mejores examenes ana. tomopatológicos y reacciones biológi cas seriadas, en todos aquellos casos en que la sintomatologia acuse un diagnóstico probable de corioepite. lioma.

La historia relatada es tánto más elocuente cuanto que ella se produjo con embarazo que evolucionó normalmente hasta culminar en parto a término, realizado en forma espontánea. sin que al examen macroscópico del feto o de los anexos se hallara nada anormal. Cuántos abortos molares no sufrirán la misma transformación ma. ligna de sus elementos sincitiales, si se tiene en cuenta que es alli, en ese tejido de proliferación exagerada, donde se inicia la degeneración maligna? Pero es que, en verdad, para el diagnóstico exacto del corio-epitelioma se requiere su estudio anatomo-patológi$\mathrm{co}$, y en muy pocas ocasiones se practicer.

\section{JUAN N, BAQUERO}

Se hace cargo de la Administración de Revistas de carácter científico.

Calle 16, númeno 7-91. Oficina 4. Teléfono 23-901.

Apartado Nacional 276. Bogotá. 